\title{
Enhanced Visual Attentional Modulation in Patients with Inherited Peripheral Retinal Degeneration in the Absence of Cortical Degeneration
}

\author{
Sónia Ferreira $\mathbb{D}^{1},{ }^{1}$ Andreia Carvalho Pereira, ${ }^{1,2}$ Bruno Quendera, ${ }^{3}$ Aldina Reis $\left(\mathbb{D},{ }^{1,4}\right.$ \\ Eduardo Duarte Silva, ${ }^{1}$ and Miguel Castelo-Branco $\mathbb{D}^{1,3}$ \\ ${ }^{1}$ Coimbra Institute for Biomedical Imaging and Translational Research, CIBIT, ICNAS-P, CNC.IBILI, Faculty of Medicine, \\ University of Coimbra, 3000-548 Coimbra, Portugal \\ ${ }^{2}$ Sackler Institute for Translational Neurodevelopment, Department of Forensic and Neurodevelopmental Science, \\ Institute of Psychiatry, Psychology, and Neuroscience, King's College London, UK \\ ${ }^{3}$ CNC.IBILI, Institute of Nuclear Sciences Applied to Health, ICNAS, University of Coimbra, 3000-548 Coimbra, Portugal \\ ${ }^{4}$ Ophthalmology Unit, Centro Hospitalar e Universitário de Coimbra, 3000-075 Coimbra, Portugal
}

Correspondence should be addressed to Miguel Castelo-Branco; mcbranco@fmed.uc.pt

Received 3 February 2019; Accepted 11 June 2019; Published 25 June 2019

Academic Editor: Stuart C. Mangel

Copyright (C) 2019 Sónia Ferreira et al. This is an open access article distributed under the Creative Commons Attribution License, which permits unrestricted use, distribution, and reproduction in any medium, provided the original work is properly cited.

\begin{abstract}
The role of attentional mechanisms in peripheral vision loss remains an outstanding question. Our study was aimed at determining the effect of genetically determined peripheral retinal dystrophy caused by Retinitis Pigmentosa (RP) on visual cortical function and tested the recruitment of attentional mechanisms using functional magnetic resonance imaging (fMRI). We included thirteen patients and twenty-two age- and gender-matched controls. We analyzed cortical responses under attentional demands and passive viewing conditions while presenting a visual stimulus covering the central and paracentral visual field. Brain activity was studied in visual areas V1, V2, and V3 as well as in cortical regions of interest corresponding to the preserved and the damaged visual field. The influence of visual field extent and age of disease onset were also investigated. Cortical thickness of visual areas was also measured. We found that cortical visual responses under attentional demands were increased in patients with larger degeneration of visual field, as demonstrated by significant interaction effects between group and task conditions. Moreover, activation during the task condition was increased for patients in two cortical regions of interest corresponding to the preserved and damaged visual field, specifically in patients with severe visual field loss. These findings were observed in the presence of preserved visual cortical structure. We conclude that RP patients have enhanced visual attention recruitment despite their retinal degeneration, while cortical structure and overall response levels remain intact. The unmasking of feedback signals from higher level visual regions involved in attentional processes may explain the increased cortical responses. These findings are relevant for the design of strategies for treating retinal diseases, based on attentional cuing.
\end{abstract}

\section{Introduction}

Human studies concerning the effects of peripheral retinal loss on adult visual cortical structure and function are scarce. Previous studies have mainly addressed central retinal disorders such as macular degeneration or other hereditary retinal dystrophies and diseases such as glaucoma [1-3]. Peripheral and central visual information is differentially routed in the brain $[4,5]$. Thus, neural adaptation mechanisms might differ when central or peripheral visual degeneration occurs. Previously, we found evidence for visual retinotopic reorganization in RP (peripheral regions responding to more central representations) [6]. It is nevertheless also important to assess the impact of peripheral retinal loss on visual 
attentional mechanisms. This may provide useful information in the context of low and high level strategies for treating different retinal diseases. Additionally, there is still an ongoing debate on the nature of adult brain functional reorganization induced by retinal diseases [7-10].

Our study is aimed at determining the interaction between attentional mechanisms and peripheral retinal dystrophy caused by Retinitis Pigmentosa (RP) on brain function using magnetic resonance imaging (MRI). RP is an inherited degeneration of photoreceptors that initially affects the peripheral retina and later advancing towards the central retina. The onset age varies from infancy to adulthood. The disease manifestations comprise night blindness, tunnel vision, and possibly blindness in severe stages $[11,12]$. In a functional magnetic resonance imaging (fMRI) case series study of three RP patients [13], authors reported taskdependent changes in cortical responses in the lesion projection zone (LPZ-the cortical region that no longer receives input due to a bilateral retinal lesion or scotomata [1]). They suggested the unmasking of feedback signals from higherorder visual areas under attentional demands when retinal input signals are lost. Another fMRI report with one RP patient found no evidence of functional alterations in the LPZ [14]. Our recent study in a relatively large cohort showed clear topological evidence for reorganization, which was dependent on the long-term extent of visual loss [6].

Contrasting with RP, central vision is primarily affected in macular degeneration. Some authors claimed that the deafferented cortical neurons in the primary visual cortex become responsive to inputs from the peripheral retina in this pathology [15-20]. However, other studies have questioned such visual cortical alterations by reporting the existence of a silent LPZ [14, 21-25]. Some of the previous studies showed that visual cortical alterations in macular degeneration are associated with the severity of retinal function loss, arguing that large-scale reorganization only occurs when there is a complete foveal visual loss $[15,18,20]$. However, other researchers did not find any signs for cortical reorganization in a large cohort of patients without foveal sparing [22]. Moreover, the influence of age of disease onset on the degree of cortical alterations is not clear $[15,22]$, although there is some evidence for larger reorganization in macular degeneration patients with earlier forms of the disease [19]. The reduced numbers of participants [21, 25] and the difference in stimuli and tasks used [26] may have also contributed to the controversy in the reported macular degeneration studies ([1], [7].

Our study was aimed at investigating the effect of peripheral retinal dystrophy caused by RP on brain attentional mechanisms using fMRI taking into account the effect of visual field extent and age of disease onset. Our hypothesis stated that, in addition to the previously demonstrated reorganization, visual cortical responses were also altered as a function of attentional demands in RP patients due to the lack of peripheral retinal bottom-up input. Moreover, we hypothesized that these alterations were more prominent for RP patients with more constricted visual fields and earlier disease onset.

\section{Methods}

2.1. Participants. The participants selected for this study were also included in a previous work from our group on visual retinotopy [6]. We included $13 \mathrm{RP}$ individuals (8 males and 5 females; mean age $38.31 \pm 12.65$ years; age range $20-66$ years; 12 right-handed and 1 left-handed; self-reported symptomatic age of onset range 2 - 39 years, resulting in symptomatic duration range 6 - 42 years) and 22 control subjects ( 11 males and 11 females; mean age $38.45 \pm 12.29$ years; age range 23 - 66 years; 21 right-handed and 1 left-handed). Both groups were matched for age $\left(t_{(33)}=-0.03\right.$, nonsignificant $p>0.050(\mathrm{NS}))$, gender $\left(\chi_{(1)}^{2}=0.44, \mathrm{NS}\right)$, and handedness $\left(\chi_{(1)}^{2}=0.15\right.$, NS) ratio. Patients were recruited at the Centro Hospitalar e Universitário de Coimbra. The control group participants were local volunteers. The study was conducted in accordance with the Declaration of Helsinki and was approved by the Ethics Committee of Faculty of Medicine of the University of Coimbra. Written informed consent was obtained from all participants. Exclusion criteria were intracranial abnormalities, movement during MRI acquisitions, fixation instability, visual alterations in control subjects, or visual alterations other than RP for patients (e.g., diabetic retinopathy or glaucoma).

2.2. Ophthalmological Assessment. For each participant, we measured visual acuity with a decimal chart (converted to logarithm of Minimum Angle of Resolution (logMAR) scale), average cortical thickness, and retinal nerve fiber layer (RNFL) thickness with Frequency Domain Cirrus Ocular Coherence Tomography (OCT, software version 5.1.1.6, Carl Zeiss Meditec AG, USA), and static visual fields with a MonCv3 multifunction perimeter (Metrovision, France) (Figure 1). A detailed description of the methodology used was described in our previous study [6] (Table 1).

2.3. Brain Imaging Procedures. Scanning was performed on a $3 \mathrm{~T}$ scanner (Magneton TrioTim, Siemens AG, Germany) at the Portuguese Brain Imaging Network, using a 12channel birdcage head coil. Two anatomical T1-weighted Magnetization-Prepared Rapid Acquisition with Gradient Echo (MPRAGE) sequences with $1 \times 1 \times 1 \mathrm{~mm}^{3}$ voxel size, Repetition Time (TR) $2.53 \mathrm{~s}$, Echo Time (TE) $3.42 \mathrm{~ms}$, Flip Angle (FA) $7^{\circ}$, Field Of View (FOV) $256 \times 256 \mathrm{~mm}^{2}$, and 176 slices were acquired from each participant. Functional sequences consisted of a single shot Echo-Planar Imaging (EPI) acquired in the axial plane parallel to the Anterior Commissure (AC)-Posterior Commissure (PC) plane with $2 \times 2 \times 2 \mathrm{~mm}^{3}$ voxel size, TR $2 \mathrm{~s}$, TE $39 \mathrm{~ms}$, interslice time (TI) $76 \mathrm{~ms}$, FA $90^{\circ}$, FOV $256 \times 256 \mathrm{~mm}^{2}, 26$ slices, and $128 \times 128$ imaging matrix.

Stimuli were presented using MRI compatible goggles with refractive correction (VisualSystem, NordicNeurolab, Norway). One eye was covered with a cotton patch while the other received the visual input (the dominant eye, except if it was the eye with the worst visual acuity). The RP and control group were matched for the selected eye ratio $\left(\chi_{(1)}^{2}=0.85\right.$, NS) and for the dominance of the 
RP 2 (LE $8.0 \mathrm{deg})$

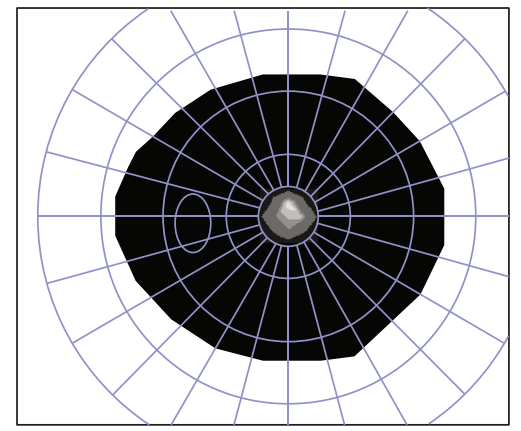

RP 7 (LE 14.5 deg)

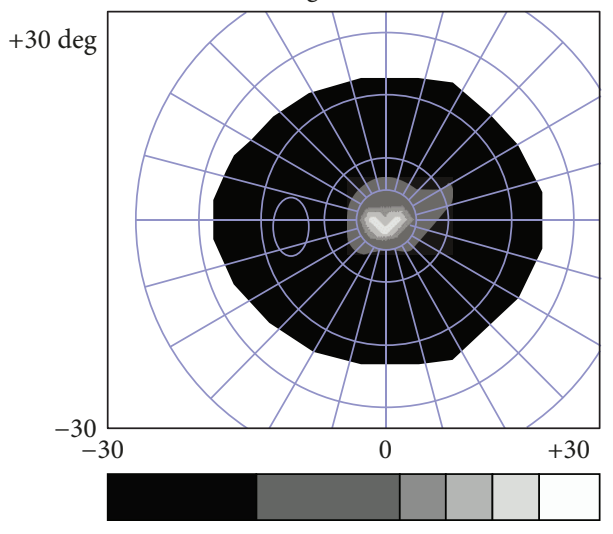

0
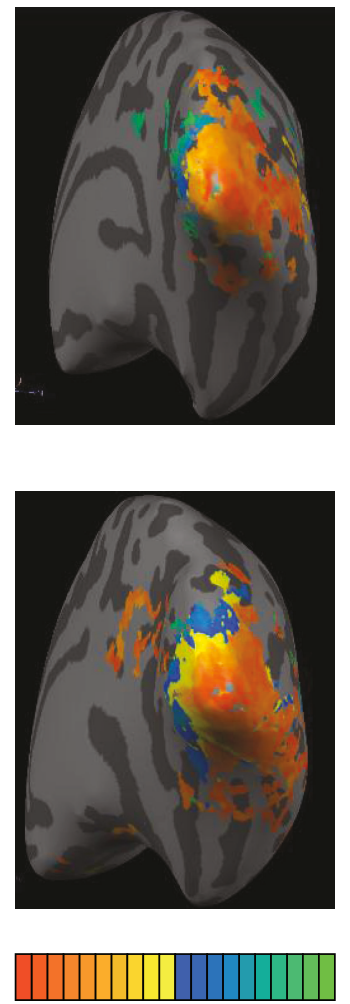

FIGURE 1: Representation of the left eye lesions (scotomata) measured with static perimetry on the left side of the figure (gray scale represents visual field sensitivity in $\mathrm{dB}$ ), and right hemisphere retinotopic eccentricity maps on the right side of the figure (colored axis represents visual field extent in degrees ( 1 to $23 \mathrm{deg}$ ); Linear Correlation Maps, $r>0.25$; inflated hemisphere mesh) in two patients. RP $=$ Retinitis Pigmentosa and LE $=$ left eye.

selected eye ratio $\left(\chi_{(1)}^{2}=0.01\right.$, NS; 1 patient with missing data). The maximum field of view was $23 \times 30 \mathrm{deg}$ (resolution of $600 \times 800)$.

2.4. MRI Stimuli. Stimuli were designed using Matlab 2011b (the MathWorks Inc., USA) with Psychophysics Toolbox 3 extensions (http://psychtoolbox.org/). A central red-colored cross with $0.78 \mathrm{deg}$ of diameter was used for fixation [6].

2.4.1. Retinotopic Mapping Stimuli. Polar angle and eccentricity stimuli were employed to delineate the cortical visual areas (V1, V2, and V3) using the traveling-wave approach from the standard phase-encoded retinotopic mapping [27]. Polar angle maps were obtained using a black and white flickering checkerboard wedge with $45 \mathrm{deg}$ rotating in an anticlockwise direction (initial angle of $22.50 \mathrm{deg}$ with horizontal axis). Eccentricity maps were obtained using a black and white flickering checkerboard expanding ring (for additional details see [6]). Checkerboard size varied with cortical magnification factor from the center to the periphery $[28,29]$. Stimuli flickering frequency was $8 \mathrm{~Hz}$ and contrast was $\sim 100 \%$. Each run comprised 2 baseline blocks ( $0 \%$ contrast; $12 \mathrm{~s}$ ) at the beginning and end of the run with 4 cycles of polar angle or eccentricity stimuli (48 s each; total duration of a run 216s). Two runs of polar angle and 2 runs of eccentricity were acquired for each subject.

2.4.2. Attentional Task Stimuli. The set of stimuli of the main experimental task of this study consisted of a random sequence of 2 different sized checkerboard rings pseudorandomly presented during either passive viewing (fixation only) or a one-back visual memory task condition. During the task condition, participants were instructed to press a button every time a ring was the same size as the immediately preceding one. Ring 1 was presented at a foveal location (diameter between 0.78 and $1.90 \mathrm{deg}$ ), and Ring $_{2}$ at a parafoveal location of the visual field (diameter between 6.74 and $9.52 \mathrm{deg}$ ). Ring thickness varied with the cortical magnification factor from the center to the periphery [28, 29]. Rings appeared during $0.50 \mathrm{~s}$ in random intervals of $1.50,3.50$, or $5.50 \mathrm{~s}$ within each block. Four passive viewing blocks and 4 task blocks ( $\sim 100 \%$ contrast; flickering frequency of $8 \mathrm{~Hz}$; 10 rings; $36 \mathrm{~s}$ ) were alternately presented, intercalated with 9 baseline blocks ( $\sim \%$ contrast; $12 \mathrm{~s}$; each run began and ended with one baseline block). Auditory instructions were provided to the subject before each block, depending on the condition: "Rest" for passive viewing or "Answer" for the one-back task. The average response time and percentage of errors during the task were recorded. Participants who 


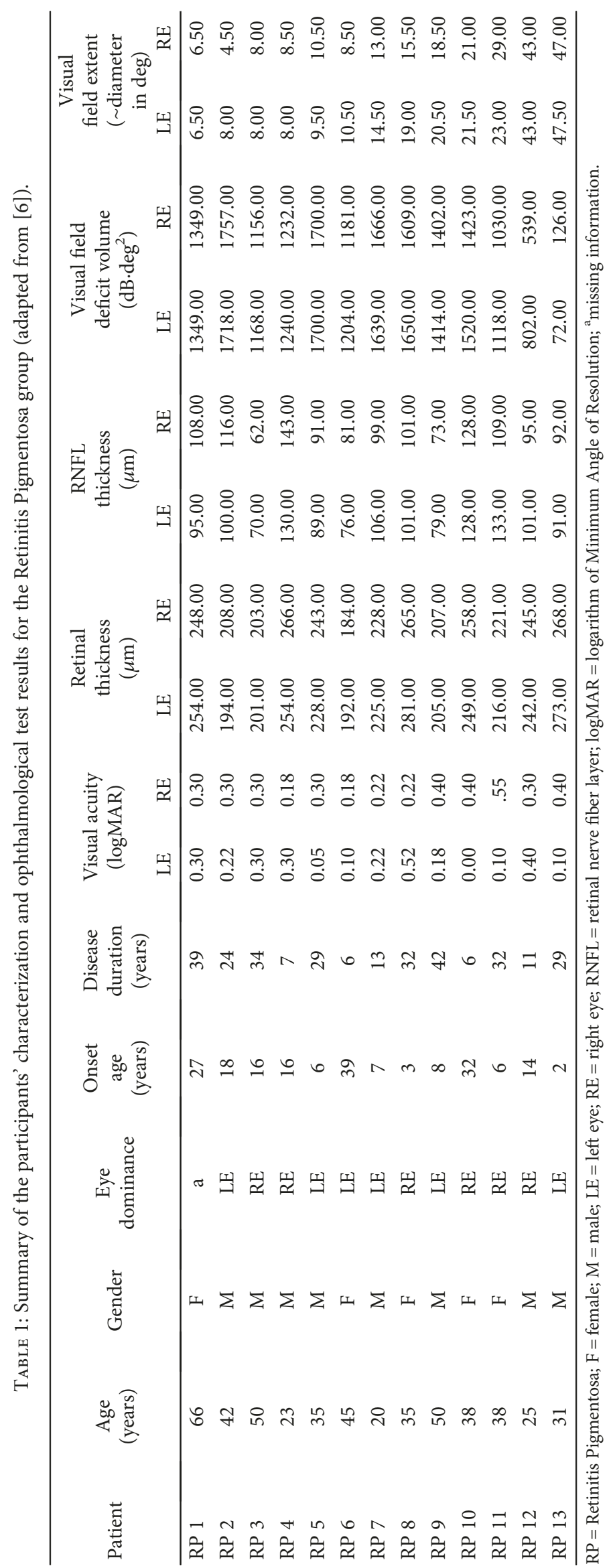




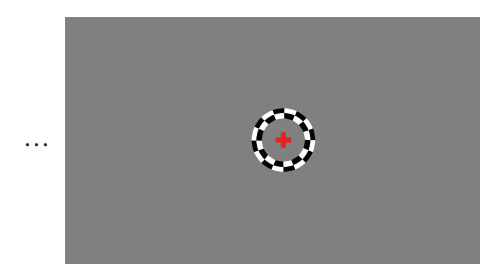

Ring $1(0.5 \mathrm{~s})$

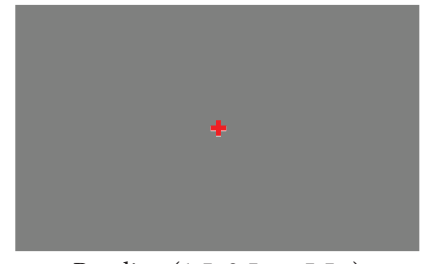

Baseline $(1.5,3.5$, or $5.5 \mathrm{~s})$

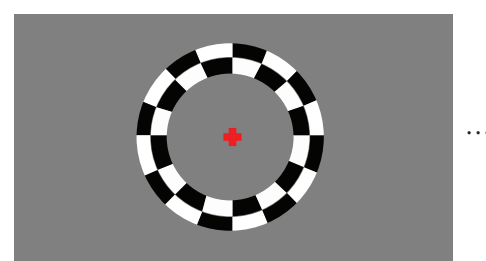

Ring $2(0.5 \mathrm{~s})$

FIGURE 2: Representation of the task paradigm with the central (Ring 1 ) and the paracentral $\left(\operatorname{Ring}_{2}\right)$ flickering checkerboard rings and the interstimulus intervals. During the passive viewing condition, participants had to fixate the central red cross. During the visual memory task condition, participants pressed a button every time a repeated ring appeared (one-back task). Scale of the fixation dot has been changed to enhance visibility.

answered during passive viewing blocks were excluded. Figure 2 shows a representation of the one-back task stimulus. We aimed at performance matching between patients and controls. We preferred this choice as compared to a possible 2-back task, because we believe that this would lead to quite large executive load.

2.5. MRI Data Processing. Brain imaging analysis was performed with BrainVoyager QX 2.6.1 (Brain Innovation B. V., Netherlands). The two anatomical images of each participant were averaged, reoriented to AC-PC plane, and transformed to Talairach (TAL) space. The image was segmented into cerebral spinal fluid, gray matter, and white matter to create inflated mesh representations of each hemisphere. A cut was manually drawn along the calcarine sulcus, and meshes were flattened for retinotopic maps projection $[6,30]$.

The preprocessing of functional sequences consisted of scan time correction, temporal high-pass filtering ( 2 cycles per run), spatial smoothing (FWHM $2 \mathrm{~mm}$ ), and a correction for small interscan head movements. Participants were excluded if within-run movements exceeded $4 \mathrm{~mm}(-2$ to $2 \mathrm{~mm}$ ). Polar angle and eccentricity maps were obtained from the average of the two runs, created based on linear regression analysis, and projected onto the flattened surfaces of each subject (statistical maps with $r>0.25$; Figure 1) [6]. Field Sign Maps were automatically created using polar angle and eccentricity Look-Up Table maps. Retinotopic areas V1 dorsal $\left(\mathrm{V} 1_{\mathrm{d}}\right), \mathrm{V} 1$ ventral $\left(\mathrm{V} 1_{\mathrm{v}}\right), \mathrm{V} 2$ dorsal $\left(\mathrm{V} 2_{\mathrm{d}}\right)$, $\mathrm{V} 2$ ventral $\left(\mathrm{V} 2_{\mathrm{v}}\right)$, $\mathrm{V} 3$ dorsal $\left(\mathrm{V}_{\mathrm{d}}\right)$, and $\mathrm{V} 3$ ventral $\left(\mathrm{V}_{\mathrm{v}}\right)$ were manually defined for each subject in each hemisphere on flattened meshes.

Statistical analyses were performed on individual data in TAL space using a general linear model (GLM) $(z$-transformation, False Discovery Rate (FDR) $q<0.05$, correction for temporal serial correlations $\mathrm{AR}(2))$ within the retinotopically defined visual areas. Response predictors for the visual memory task were obtained, and beta values evoked by each stimulus conditions were retrieved: Ring Passive Viewing, Ring $_{2}$ Passive Viewing, Ring ${ }_{1}$ Task, and Ring 2 Task. Figure 3 represents the visual cortical responses for all predictors during the visual memory task for RP patients and control participants.

A multistudy GLM (random fixed effects, $z$-transformation, FDR $q<0.05, \operatorname{AR}(2))$ was also run in two different regions of interest along the calcarine sulcus (V1). The func- tional projection zone (FPZ) represented the preserved visual field region, and the LPZ represented either the visual field scotomata in patients or the unstimulated visual field in controls. These cortical regions were manually defined considering the retinotopic eccentricity maps of each participant. Figure 4 illustrates the location of the two regions of interest, the FPZ and the LPZ, in one control participant. Response predictors for the visual working memory task were obtained, and beta values evoked by each stimulus conditions were retrieved inside these regions of interest: Ring 1 Passive Viewing, Ring 2 Passive Viewing, Ring $_{1}$ Task, and Ring 2 Task.

Cortical thickness was calculated on the retinotopic areas using the standard procedure of BrainVoyager (see [30] for a complete description). To allow an accurate segmentation of white matter-gray matter and gray matter-cerebral spinal fluid boundary, TAL anatomical data were converted to high-resolution $0.5 \times 0.5 \times 0.5 \mathrm{~mm}^{3}$. The subcortical structures and the ventricles were filled as white matter. After computation, cortical thickness maps were superimposed on cortical meshes, and mean cortical thickness values of all visual areas were extracted using Matlab BVQXtools toolbox extensions (http://support.brainvoyager.com/available-tools/ 52-matlab-tools-bvxqtools.html).

2.6. Subgroup Analysis. To understand the influence of the level of peripheral degeneration and disease onset age on visual cortex response, the RP group was divided accordingly to these factors. The RP group was divided accordingly to the extent of visual field measured by the static perimetry test in two subgroups: RPsvf (small visual field) $(n=6$, patients RP 1 to RP 6) with bilateral visual field diameter under 9.52 deg and RPlvf (large visual field) ( $n=7$, patients RP 7 to RP 13) with bilateral visual field diameter over $9.52 \mathrm{deg}$ (see Table 1). In this way, the RPlvf patients were expected to see the complete visual working memory task stimuli $\left(\operatorname{Ring}_{1}\right.$ and Ring ${ }_{2}$, because the maximum diameter of Ring 2 was $9.52 \mathrm{deg}$ ), whereas most RPsvf patients would only partially see Ring ${ }_{2}$. Both subgroups and the control group were matched for age $\left(F_{(2,32)}=1.00, \mathrm{NS}\right)$, gender $\left(\chi_{(2)}^{2}=0.56, \mathrm{NS}\right)$, handedness $\left(\chi_{(2)}^{2}=1.37, \mathrm{NS}\right)$, selected eye $\left(\chi_{(2)}^{2}=1.44, \mathrm{NS}\right)$, and selected eye dominance ratio $\left(\chi_{(2)}^{2}=1.65\right.$, NS; 1 patient with missing data). Additionally, the disease onset age $\left(F_{(1,11)}=2.80, \mathrm{NS}\right)$ 


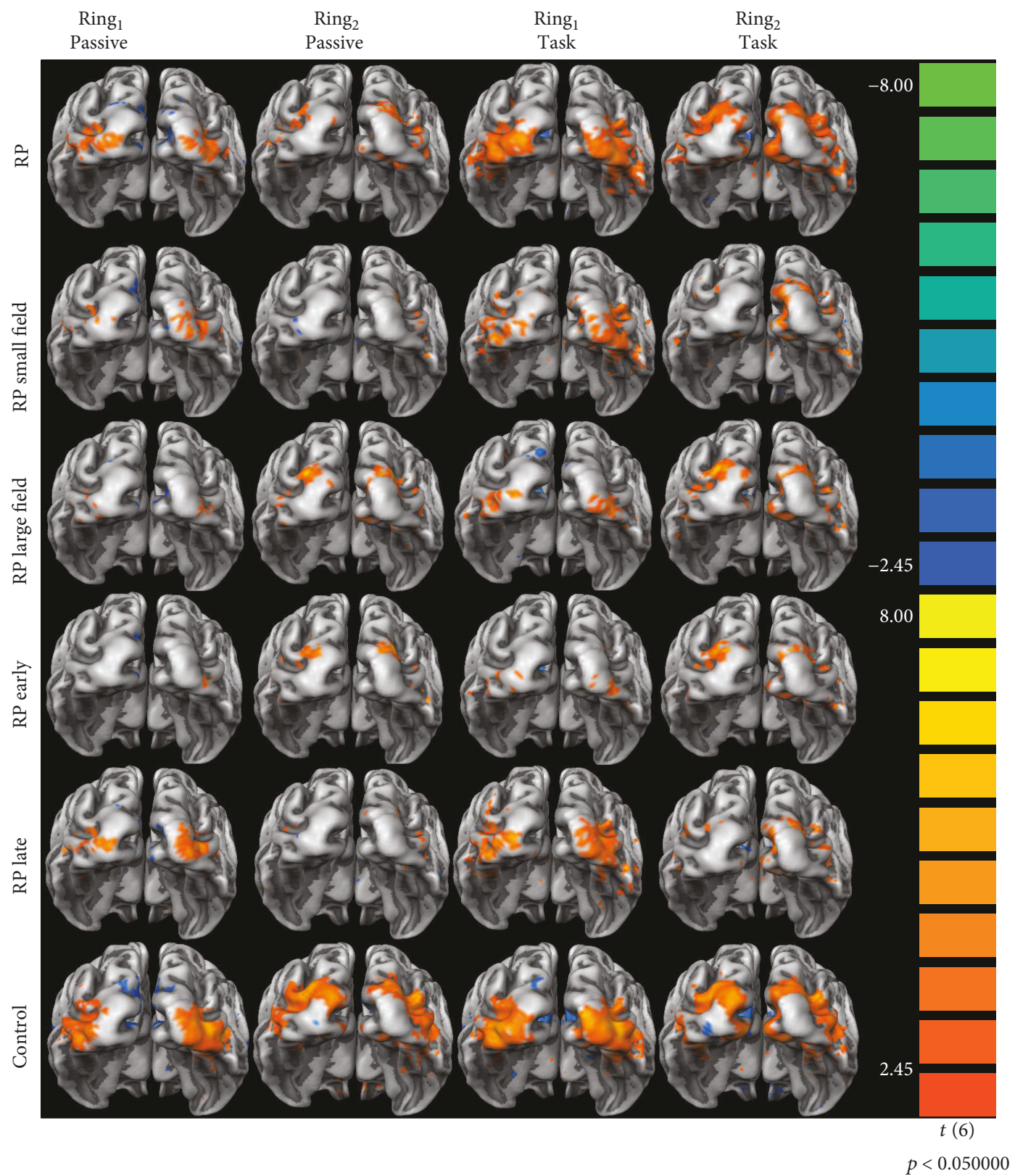

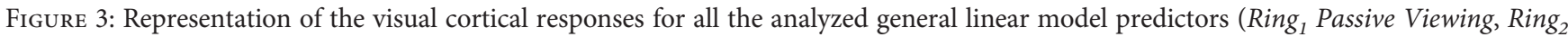
Passive Viewing, Ring ${ }_{1}$ Task, and Ring 2 Task) for the Retinitis Pigmentosa group (RP) and the control group. The visual cortical activation is also represented for the subgroups of patients RPsvf (RP small field) with less than 9.52 deg of visual field diameter and RPlvf (RP large field) with more than $9.52 \mathrm{deg}$ of visual field diameter. Finally, the visual cortical responses are also displayed for the subgroups of patients RPeo (RP early) with disease onset ages lower than 14 years and RPlo (RP late) with onset ages higher or equal to 14 years. Images represented the posterior view of both hemispheres meshes averaged for all participants. The colored scale represents the $t$-test value for the contrast predictor versus baseline with $p<0.050$

and the disease symptomatic duration $\left(F_{(1,11)}=0.00\right.$, NS) were not different between the subgroups RPsvf and RPlvf.

The RP group was also divided accordingly to the disease onset age into two subgroups: RPeo (early onset) $(n=6$, patients RP 5, 7, 8, 9, 11, and 13) with an onset age lower than 14 years and RPlo (late onset) ( $n=7$, patients RP $1,2,3,4,6$, 10 , and 12) with an onset age greater than or equal to 14 years (see Table 1). Patients from subgroup RPeo with earlier disease onset age were expected to have more prominent alterations in visual cortex responses than RPlo patients since some visual plasticity is thought to remain until 14 to 16 years of age $[8,31]$. Both subgroups and the control group were matched for age $\left(F_{(2,32)}=0.43\right.$, NS), gender $\left(\chi_{(2)}^{2}=0.57, \mathrm{NS}\right)$, handedness $\left(\chi_{(2)}^{2}=1.82\right.$, NS $)$, selected eye $\left(\chi_{(2)}^{2}=2.96\right.$, NS), and selected eye dominance ratio $\left(\chi_{(2)}^{2}=0.01\right.$, NS; 1 patient with missing data). As expected, the disease onset age $\left(F_{(1,11)}=19.29, p=0.001\right)$ was different 


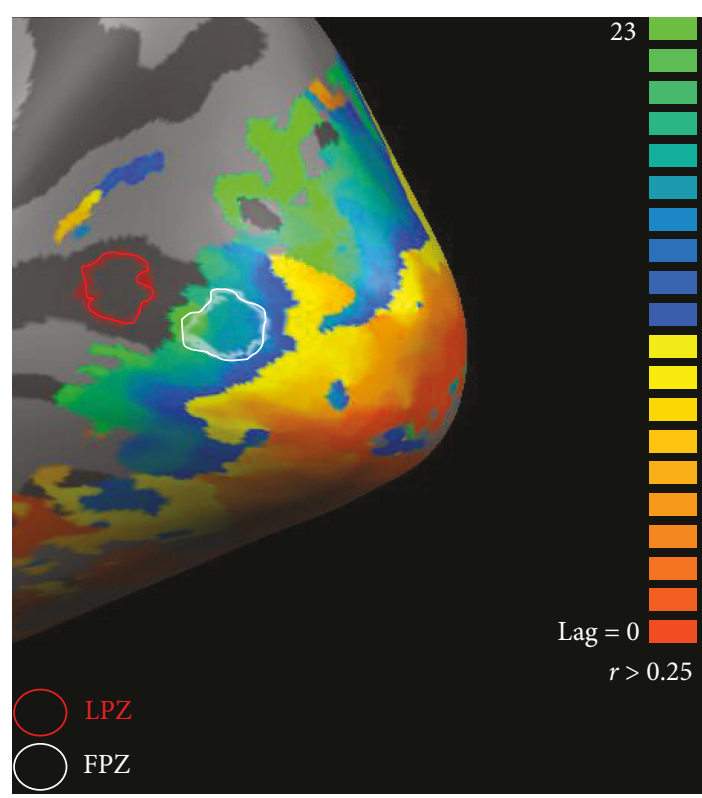

Figure 4: Representation of the cortical regions of interest-the function projection zone (FPZ) and lesion projection zone (LPZ) - on the right hemisphere of a control participant. FPZ represents the preserved visual field region and the LPZ represents the visual field scotomata in patients or the unstimulated visual field in controls. These cortical regions were manually defined along the calcarine sulcus (V1) considering the retinotopic eccentricity map.

between the two subgroups of patients RPeo and RPlo, but the disease symptom duration $\left(F_{(1,11)}=2.81\right.$, NS) was not different. Table 2 summarizes the information of visual field extent and onset age for all subgroups.

2.7. Statistical Analysis. Statistical analysis was performed with IBM SPSS Statistics, Version 22 (IBM Corporation, USA). Normality and homogeneity of variance were tested using Shapiro-Wilk's test and Levene's test, respectively. For data in accordance with these assumptions, statistical parametric tests were performed. Otherwise, nonparametric methods were applied. Bonferroni correction was applied for multiple comparisons ( $p$ values presented as corrected $p$ ). The epsilon value was used for correction of nonspherical data (Huynh-Feldt for epsilon higher than 0.75 and Greenhouse-Geisser for epsilon lower than 0.75). The significance level was 0.05 , and the statistical power was higher than 0.80 for all presented results.

\section{Results}

3.1. Visual Assessment. The first two parts of the results section (Visual Assessment and Behavioral Data) are focused on ophthalmological features-visual acuity, visual field extent, average retinal thickness, RNFL thickness-, and performance in visual memory task in patient and clinical groups and subgroups. The subsequent parts are focused on the main hypotheses of the article.
3.1.1. All Groups (No Stratification according to Visual Field Extent or Age of Onset). Visual acuity (left eye (LE) $U=$ $275.00, p=2.642 \times 10^{-7}$ and right eye (RE) $U=286.00, p=$ $1.355 \times 10^{-9}$ ) and average retinal thickness (LE $U=275.00$, $p=1.883 \times 10^{-7}$ and RE $U=283.00, p=9.483 \times 10^{-9}$ ) were reduced in both eyes for patients as compared to control participants. Visual field deficit volume (LE $U=3.00, p=$ $9.483 \times 10^{-9}$ and RE $\left.U=2.00, p=5.419 \times 10^{-9}\right)$ was higher in both eyes for patients as compared to the control group. No differences were found for RNFL thickness in both eyes between the two groups (LE $U=125.00$, NS and RE $U=120.00$, NS). Moreover, no statistically significant differences were found between the left and right eyes within groups for visual acuity (RP $Z=1.33$, NS and control $Z=1.41, \mathrm{NS}$ ), average retinal thickness (RP $Z=-0.84$, NS and control $Z=-0.88$, NS), RNFL thickness (RP $Z=0.00$, NS and control $Z=-0.07$, NS), visual field deficit volume (RP $Z=-1.24$, NS and control $Z=$ -0.28 , NS), and visual field extent (RP $Z=-1.18$, NS; controls have a constant visual field extent equal to $48 \mathrm{deg}$ corresponding to the maximum diameter covered by our static perimetry) [6].

3.1.2. Visual Field (Large vs. Small Visual Field) Subgroup Analysis. Visual acuity $\left(\mathrm{LE} \chi_{(2)}^{2}=22.62, p=1.200 \times 10^{-5}\right.$ and $\left.\operatorname{RE} \chi_{(2)}^{2}=25.64, p=3.000 \times 10^{-6}\right)$ and average retinal thickness $\left(\operatorname{LE} \chi_{(2)}^{2}=20.91, p=2.900 \times 10^{-5}\right.$ and $\operatorname{RE} \chi_{(2)}^{2}=$ 23.01, $p=1.000 \times 10^{-5}$ ) were reduced in both eyes for the two RP subgroups as compared to control participants. No differences were found for RNFL thickness in both eyes between the three groups ( $\mathrm{LE} \chi_{(2)}^{2}=3.00$, NS and RE $\chi_{(2)}^{2}=0.65$, NS). Visual field deficit volume $\left(\operatorname{LE} \chi_{(2)}^{2}=\right.$ 23.00, $p=1.000 \times 10^{-5}$ and $\operatorname{RE} \chi_{(2)}^{2}=23.29, p=9.000 \times$ $10^{-6}$ ) was higher in both eyes for both patients' subgroups as compared to the control group. According to the subgroup division, visual field extent was different between the two subgroups ( $\mathrm{LE} \chi_{(1)}^{2}=9.10, p=0.003$ and $\mathrm{RE}$ $\chi_{(1)}^{2}=9.02, p=0.003$ ), with RPsvf (small visual field) patients presenting smaller visual fields than RPlvf (large visual field/more preserved). Moreover, no statistically significant differences were found between the left and right eyes within groups for visual acuity (RPsvf $Z=0.73$, NS, RPlvf $Z=1.26$, NS, and control $Z=1.41$, NS), average retinal thickness (RPsvf $Z=1.15$, NS, RPlvf $Z=0.42$, NS, and control $Z=0.88$, NS), RNFL thickness (RPsvf $Z=1.58$, NS, RPlvf $Z=-1.76$, NS, and control $Z=-0.07$, NS), visual field deficit volume (RPsvf $Z=-0.36$, NS, RPlvf $Z=-1.35$, NS, and control $Z=-0.28$, NS), and visual field extent (RPsvf $Z=-0.73$, NS and RPlvf $Z=-0.95$, NS; controls have a constant visual field extent equal to $48 \mathrm{deg}$ corresponding to the maximum diameter covered by static perimetry).

3.1.3. Age of Onset Subgroup Analysis. Visual acuity (LE $\chi_{(2)}^{2}=22.55, p=1.300 \times 10^{-5}$ and $\mathrm{RE} \chi_{(2)}^{2}=25.42, p=$ $\left.3.000 \times 10^{-6}\right)$ and average retinal thickness $\left(\operatorname{LE} \chi_{(2)}^{2}=\right.$ 20.77, $p=3.100 \times 10^{-5}$ and $\operatorname{RE} \chi_{(2)}^{2}=22.89, p=1.100 \times$ 


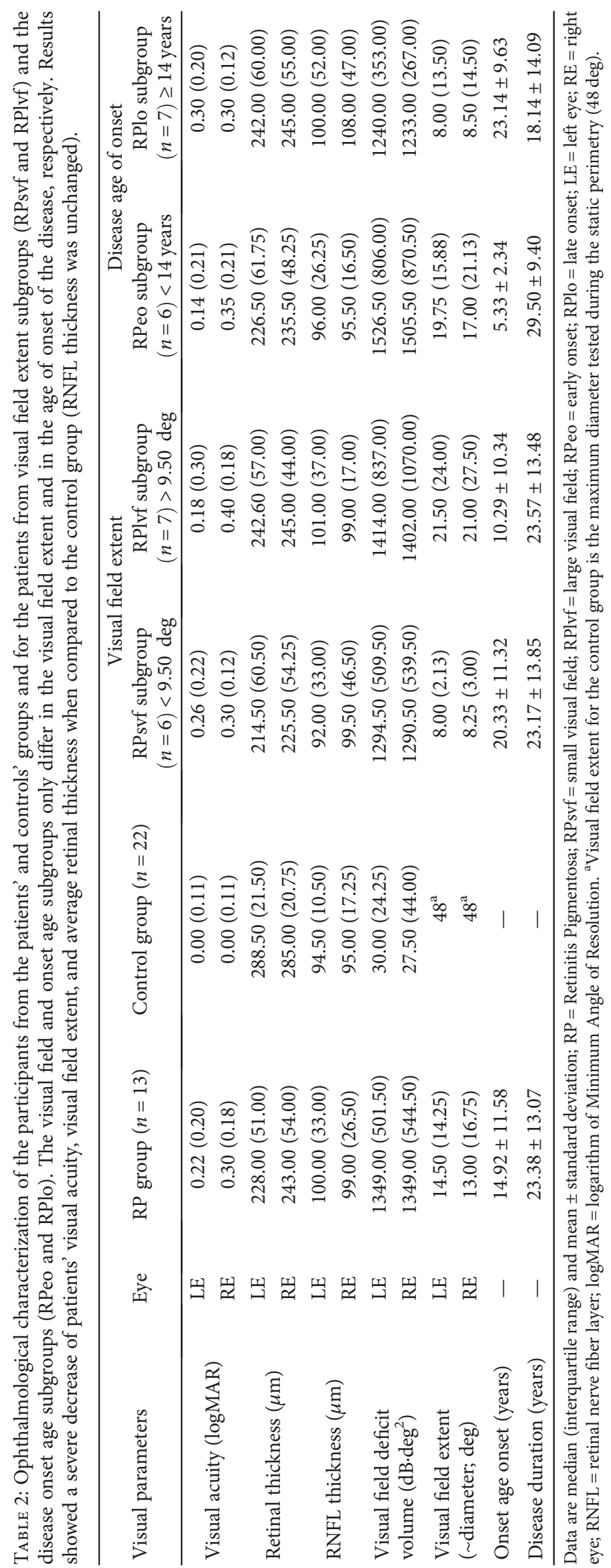


$10^{-5}$ ) were reduced in both eyes for the two RP subgroups (RPeo, early onset; RPlo, late onset) as compared to control participants. No differences were found for RNFL thickness in both eyes between the three groups $\left(\operatorname{LE} \chi_{(2)}^{2}=\right.$ 0.38 , NS and $\operatorname{RE} \chi_{(2)}^{2}=1.48$, NS). Visual field deficit volume $\left(\operatorname{LE} \chi_{(2)}^{2}=22.88, p=1.100 \times 10^{-5}\right.$ and $\operatorname{RE} \chi_{(2)}^{2}=$ 23.19, $p=9.000 \times 10^{-6}$ ) was higher in both eyes for both patients' subgroups as compared to the control group. The visual field extent was not different between the two subgroups of patients ( $\mathrm{LE} \chi_{(1)}^{2}=2.50$, NS and RE $\chi_{(1)}^{2}=2.95$, NS). Moreover, no statistically significant differences were found between the left and right eyes within groups for visual acuity (RPeo $Z=1.08$, NS, RPlo $Z=0.13$, NS, and control $Z=1.41$, NS), average retinal thickness (RPeo $Z=-0.21$, NS, RPlo $Z=-1.18$, NS, and control $Z=-0.88$, NS), RNFL thickness (RPeo $Z=-1.21$, NS, RPlo $Z=-1.16$, NS, and control $Z=-0.07$, NS), visual field deficit volume (RPeo $Z=-0.40$, NS, RPlo $Z=-1.36$, NS, and control $Z=-0.28, \mathrm{NS}$ ), and visual field extent (RPeo $Z=-0.52$, NS and RPlo $Z=-1.29$, NS; controls have a constant visual field extent equal to 48 deg corresponding to the maximum diameter covered by static perimetry).

In sum, ophthalmological tests showed decreased patients' visual acuity, visual field extent, and average retinal thickness, while RNFL thickness was preserved. Importantly, subgroups defined by visual field extent and age of onset only differ in the visual field extent or in the age of onset of the disease, respectively. Table 2 shows a summary of participants' ophthalmologic characterization, presenting the visual parameter values for each group and subgroups.

3.2. Visual Memory Task: Behavioral Data. The mean response time $(U=110.00$, NS) and response error $(U=99.00, \mathrm{NS})$ were not different between the RP and control groups during the performance of the one-back visual memory task, and both groups were actually near ceiling levels. Concerning the visual field subgroups, we did not find differences among the RPsvf and RPlvf subgroups and the control group in response time $\left(F_{(2,32)}=0.03, \mathrm{NS}\right)$ and error $\left(F_{(2,32)}=1.00, \mathrm{NS}\right)$. For the onset age subgroups, the analysis did not show differences among the RPeo and RPlo subgroups and the control group in response time $\left(F_{(2,32)}=0.02\right.$, NS $)$ and error $\left(F_{(2,32)}=0.23\right.$, NS). Groups were therefore behaviorally matched.

3.3. Visual Memory Task: Responses in Functional (FPZ) and Lesion Projection Zone (LPZ). Here, we tested the hypothesis that compensatory allocation of visual attention mechanisms do occur in patients.

3.3.1. All Group. The beta values of the task predictors (Ring Passive Viewing, Ring 2 Passive Viewing, Ring 1 Task, and Ring $_{2}$ Task) were analyzed between groups for each condition (Condition Passive Viewing and Task), each ring (Ring Ring $_{1}$ and Ring 2 ), each cortical zone (Zone FPZ and LPZ in V1, see Figure 4), and each hemisphere (Hemisphere Left and Right) with repeated measures ANOVA. We found a sig- nificant effect of the predictor beta values between groups $\left(F_{(1,33)}=4.47, p=0.042\right)$. Importantly, within-subject effects for Condition $\left(F_{(1,33)}=97.18, p=2.313 \times 10^{-11}\right)$ and Condition $\times$ Group $\left(F_{(1,33)}=9.66, p=0.004\right)$ were present. To analyze the effect of the interaction Condition $\times$ Group, we used ANOVA between groups for each condition (Passive Viewing and Task). Results showed that the cortical responses during the task were higher for RP patients when compared to the control group ( $p=0.002)$, whereas no differences were found for passive viewing condition between groups, thereby corroborating the main hypothesis.

3.3.2. Analyses by Subgroups Defined by Extent of Visual Field Loss. The statistical analysis described above was conducted to study differences among the two subgroups of patients according to the extent of visual field loss and the control group. Importantly, we found the effects for Condition $\left(F_{(1,32)}=135.11, p=5.054 \times 10^{-13}\right)$, Condition $\times$ Group $\left(F_{(2,32)}=13.86, p=4.600 \times 10^{-5}\right)$, Zone $\times$ Ring $\left(F_{(1,32)}=11.52\right.$, $p=0.002)$, and Zone $\times$ Ring $\times$ Group $\left(F_{(2,32)}=6.86, p=0.003\right)$. ANOVA for each condition (Passive Viewing and Task) was applied to analyze the interaction effect of Condition $\times$ Group. Results showed that the cortical responses for task were higher for RPsvf (small visual field) patients when compared to the RPlvf (large visual field) patients (corrected $p=0.013$ ) and the control group (corrected $p=$ $\left.8.500 \times 10^{-5}\right)$, whereas no differences were found for passive viewing condition among the three groups. In this way, patients with smaller intact visual fields presented higher cortical response during the task than patients with larger visual field extent and controls. To study the effect of the interaction Zone $\times$ Ring $\times$ Group, we used ANOVA among groups for values of the ring ( $\operatorname{Ring}_{1}$ and $\operatorname{Ring}_{2}$ ) in each cortical zone (FPZ and LPZ). We found increased cortical responses for RPsvf patients for Ring ${ }_{2}$ in the FPZ when compared to controls (corrected $p=0.014$ ). In this way, patients with smaller visual fields presented higher cortical response $(2.37 \pm 2.21)$ than controls $(-0.72 \pm 2.21)$ in the FPZ during the stimulation of paracentral visual field, while patients with larger visual field extent $(0.06 \pm 2.21)$ had similar activation to controls. These observations suggest that larger damage (present in small visual field patients) lead to larger allocation of visual attention mechanisms.

3.3.3. Analyses Based on Age of Onset Defined Subgroups. The statistical analysis described above was conducted to study differences among the two subgroups of patients according to the age of disease onset and the control group. Effects for Condition $\left(F_{(1,32)}=90.76, p=7.325 \times 10^{-11}\right)$ and Condition $\times$ Group $\left(F_{(2,32)}=5.35, p=0.010\right)$ were present. ANOVA for each condition (Passive Viewing and Task) was applied to analyze the interaction effect of Condition $\times$ Group. Results showed that the cortical responses for the task were surprisingly higher for RPlo (later onset) patients when compared to the control group (corrected $p=0.006$ ), whereas no differences were found for passive viewing condition among the three groups. In sum, patients with later disease onset presented surprisingly higher cortical response during task than controls, suggesting that lower activation may represent 
more "efficient" brain activity patterns in early onset patients (with more prolonged disease evolution and more time to recruit mechanisms where "efficiency" dominates).

3.4. Visual Memory Task: Responses in Retinotopic Regions $(V 1, V 2$, and V3). In this section, we investigate how different retinotopic areas contribute to the observed patterns of activity (overall and according to the subgroups defined above).

3.4.1. All Group. The beta values of the task predictors ( $\operatorname{Ring}_{1}$ Passive Viewing, Ring 2 Passive Viewing, Ring 1 Task, and Ring 2 Task) were analyzed between groups for each condition (Condition Passive Viewing and Task), each ring (Ring Ring and Ring $_{2}$ ), each visual area (Area V1, V2, and V3), each cortical visual region (Region Ventral and Dorsal), and each hemisphere (Hemisphere Left and Right). We found the main effects of Area $\left(F_{(1.74,57.42)}=50.45, p=1.700 \times 10^{-12}\right)$, Condition $\left(F_{(1,33)}=44.27, p=1.427 \times 10^{-7}\right)$, and Ring $\left(F_{(1,33)}=53.81, p=2.019 \times 10^{-8}\right)$. We also found the effects of Area $\times$ Condition $\left(F_{(1.76,58.20)}=25.22, \quad p=4.532 \times 10^{-8}\right)$, Area $\times$ Region $\times$ Ring $\quad\left(F_{(1.72,56.92)}=9.12, \quad p=0.001\right), \quad$ and Area $\times$ Region $\times$ Ring $\times$ Group $\quad\left(F_{(1.72,56.92)}=5.98, \quad p=0.006\right)$. We can summarize these interactions by the change in visual cortical responses from V1 to V3 (Area) between passive viewing and the one-back task condition (Condition) and between Ring (central visual field) and Ring (paracentral visual field) (Ring) for both groups.

We then hypothesized that age of onset and extent of visual field loss could influence the effects in patients.

3.4.2. Visual Field Subgroup. The statistical analysis described above was conducted to study differences between the two subgroups of patients accordingly to the extent of visual field loss and the control group. We found the effects for Area $\quad\left(F_{(1.82,58.10)}=33.96, \quad p=5.461 \times 10^{-10}\right), \quad$ Condition $\left(F_{(1,32)}=58.91, p=9.513 \times 10^{-9}\right)$, Ring $\left(F_{(1,32)}=48.37, p=\right.$ $\left.7.057 \times 10^{-8}\right)$, Condition $\times$ Group $\left(F_{(2,32)}=8.53, p=0.001\right)$, Area $\times$ Condition $\quad\left(F_{(1.80,57.65)}=20.06, \quad p=5.902 \times 10^{-7}\right)$, Area $\times$ Hemisphere $\times$ Group $\quad\left(F_{(3.95,63.20)}=3.71, \quad p=0.009\right)$, and Area $\times$ Region $\times$ Ring $\quad\left(F_{(1.78,57.00)}=11.04, \quad p=1.590 \times\right.$ $10^{-3}$ ). An ANOVA for each condition (Passive Viewing and Task) was applied to analyze the critical interaction effect of Condition $\times$ Group. Results showed that the cortical responses for task were higher for RPsvf (small visual field) patients when compared to the control group (corrected $p=0.034$ ), whereas no differences were found for the passive viewing condition among groups. In this way, patients with smaller visual fields presented higher cortical response during task than controls, while patients with larger visual field extent showed responses that were similar to the control participants. To study the effect of the interaction Area $\times$ Hemisphere $\times$ Group, we used ANOVA between groups for each visual area (V1, V2, and V3) in each hemisphere (Left and Right). We found increased cortical responses for RPsvf patients in the right V1 when compared to controls (corrected $p=0.044$ ), but the remaining visual areas presented similar cortical activation. In this way, patients with smaller visual fields presented higher cortical response in the right primary visual cortex
$(3.17 \pm 1.93)$ when compared to controls $(0.87 \pm 1.93)$, while patients with larger visual field extent $(1.07 \pm 1.93)$ showed similar activation to controls.

3.4.3. Subgroup Defined by Distinct Age of Onset. The statistical analysis described above was conducted to study differences among the two subgroups of patients according to the age of onset and the control group. We found the effects for Area $\left(F_{(1.89,60.64)}=42.83, p=5.413 \times 10^{-12}\right)$, Condition $\left(F_{(1,32)}=40.44, p=3.853 \times 10^{-7}\right)$, Ring $\left(F_{(1,32)}=51.74\right.$, $\left.p=3.615 \times 10^{-8}\right)$, Area $\times \operatorname{Group}\left(F_{(3.79,60.64)}=4.46, p=0.004\right)$, Area $\times$ Condition $\quad\left(F_{(1.82,58.365)}=20.28, \quad p=4.572 \times 10^{-7}\right)$, Area $\times$ Hemisphere $\times$ Group $\quad\left(F_{(3.96,63.40)}=3.99, \quad p=0.006\right)$, Region $\times$ Ring $\times$ Group $\left(F_{(2,32)}=5.66, p=0.008\right)$, and Area $\times R e$ gion $\times \operatorname{Ring}\left(F_{(1.79,57.38)}=11.51, p=1.100 \times 10^{-4}\right)$.

In sum, this analysis focused on retinotopic areas that essentially mimics the findings observed for the LPZ and FPZ zones, corroborating the main hypothesis of preferential attentional allocation in patients, and distinct effects of the visual field lesion extent and age of onset.

3.5. Cortical Thickness of Visual Areas (V1, V2, and V3). Finally, we investigated whether functional changes were associated with structural alterations.

Visual cortical thickness differences were evaluated using repeated measures ANOVA with three within-subject factors (Area V1-V3, Region Ventral vs Dorsal, and Hemisphere Left Vs Right), one between-subject factor Group (RP vs. Control), with the average brain cortical thickness as a covariate to account for variability across participants. Cortical thickness of the individually defined visual areas was not different between the two groups $\left(F_{(1,32)}=0.047, \mathrm{NS}\right)$, and no withinsubject effects or interactions were found. This was also true for subgroup analyses.

\section{Discussion}

We investigated whether visual cortical responses in a disorder of peripheral vision are related to recruitment of attentional mechanisms. To test this hypothesis, we used a visual one-back task and passive viewing conditions with a visual stimulus covering the central ( $\left.\operatorname{Ring}_{1}\right)$ and paracentral ( Ring $\left._{2}\right)$ visual field in a group of RP patients $(n=13)$ with peripheral retinal loss and matched healthy controls $(n=22)$. Cortical responses were studied in visual retinotopic areas (V1, V2, and V3) and in two different regions of interest in V1: the FPZ representing the preserved visual field and the LPZ representing the visual field scotomata. To understand the influence of the level of peripheral degeneration and the disease age of onset, the analysis was further conducted for two distinct RP subgroups: subcategories defined by the extent of visual field loss (RPsvf, remaining small field, and RPlvf, remaining large field patients with bilateral visual field diameters under or over $9.50 \mathrm{deg}$, respectively), and subgroups defined by distinct ages of disease onset (RPeo, early onset, and RPlo, late onset-patients with age of onset of the disease lower or equal/greater than 14 years, respectively). 
Our results demonstrated that RP patients have overall preserved visual cortical responses under central and paracentral visual field stimulation. Visual cortical responses (V1, V2, and V3) to the visual memory task stimuli were also overall preserved. A critical interaction with task condition was however found: RP patients presented higher overall cortical responses during the task condition than control participants in FPZ and LPZ regions. This was further highlighted when extent of visual loss was taken into account.

Concerning the role of the extent of visual loss, RPsvf patients, with smaller visual fields, presented higher overall visual cortical responses during the task condition than control participants, while responses for RPlvf patients, with larger visual field extent, were similar to control participants. Additionally, RPsvf patients had significantly higher cortical activation in the right V1 when compared to controls, while responses for RPlvf patients were similar to healthy participants. In line with these results, RPsvf patients presented higher overall cortical responses during the task condition than control participants and RPlvf patients in LPZ and FPZ regions, while responses for RPlvf patients were similar to control participants. Additionally, the cortical activation in the FPZ was higher for RPsvf patients during the stimulation of the paracentral visual field $\left(\right.$ Ring $\left._{2}\right)$ when compared to the healthy subjects. Because visual stimuli were the same in both task and passive viewing conditions, these responses for RPsvf patients seem to be related to attentional demands during the one-back task. Masuda et al. [13] found a similar increase in striate cortical responses of three RP participants related to changes in task demands and suggested that unmasking of feedback signals from the extrastriate cortex occurs when retinal signals are absent. Such unmasking might come from activation of previously silent synapses [32]. These feedback signals might be associated with attention, visual imagery, and task-related visual processing [26]. In our work, enhanced attentional top-down modulation may compensate for the lack of retinal input from the peripheral visual field in RPsvf patients with greater visual field loss $[9,24,33]$. A recent study presented evidence for increased functional connectivity between afferent early visual areas and cortical regions involved in visual processing (middle occipital gyrus and superior temporal gyrus/sulcus) in RP patients, suggesting a possible compensatory mechanism for peripheral visual loss. These authors also found enhanced functional connectivity between the deafferented visual cortex and higher-order regions (inferior parietal lobe/sulcus and middle frontal gyrus) involved in top-down control, attentional processes, and multisensory integration [34].

However, in the three patients reported in Masuda et al.'s work, the increased responses during task were found in the V1 LPZ [13]. Here, we report an overall increase in cortical responses under task demands while analyzing V1, V2, V3, and V1 FPZ and LPZ for patients with more severe visual field degeneration. In this way, feedback signals might influence both striate and extrastriate visual cortex when there is a severe lack of peripheral retinal input, not being restricted to the LPZ. A recent work from our group provided evidence of functional remapping in V1 in the same group of patients studied here. This functional reorganization was also more prominent in RP patient with larger visual field damage [6].

Previous works with macular degeneration also showed increased cortical responses for V1 LPZ while patients performed a one-back task with peripheral stimulation [15-19, 24], contrary to passive viewing stimulation [19, 21-25]. Some of the authors showed that these V1 responses were higher for more severe central retinal loss without foveal sparing $[15,18]$, in accordance with our work. Recently, Plank et al. [26] reported that patients with central scotomata presented enhanced cortical activation in areas beyond the retinotopic cortex for complex images with naturalist scenes, supporting an increased top-down modulation of the deprived visual cortex. This result was further supported by the work of Sabbah et al. [34] showing increased functional connectivity between the LPZ and high-level regions in central retinal disease patients.

Several studies with glaucoma patients found reduced amplitude of cortical responses in V1 during passive viewing stimulation associated with structural damage of the optic disk, the RNFL thickness, and/or the visual field scotomata [35-39]. A recent study demonstrated reduced cortical activity within the LPZ in V1 and V2 in glaucoma patients under passive viewing [40]. Here, we did not find decreased activity during passive viewing in the cortical regions studied, which might indicate that RP patients have preserved visual cortical responses even for severe visual field damage, possibly due to a similar compensation mechanism by increased attentional modulation.

The cortical responses in visual areas were not globally significantly different among the RPeo and RPlo patients' subgroups with different onset ages and the control participants. Thus, overall visual cortical responses do not seem to be influenced by disease onset age, in contrast to the extent of visual loss. However, RPlo patients with later disease onset presented higher overall cortical responses during the task condition than control participants in FPZ and LPZ regions, while responses for RPeo patients with earlier forms of the disease were similar to control participants. This result was unexpected considering our initial hypothesis that earlier onset ages would lead to larger brain alterations. Two sorts of mechanisms might be operating: the first requiring long-term circuit modifications and, more present in early-onset patients, the second entailing stronger frontoparietal recruitment which tends to manifest more in patients with more recent changes in visual experience. There is indeed evidence showing that higher top-down modulation may indeed be stronger in participants with more recent changes in visual experience $[41,42]$. Moreover, it may reflect the fact that longer disease durations may lead to efficient compensatory mechanisms and decrease of frontoparietal activation. A second study from Rosa et al. [41, 42] shows that frontoparietal activation decreases over time as patients' vision becomes more adapted. Less fMRI activation might actually indicate "more efficient" compensation [41, 42]. Studies in macular degeneration patients did not report the effects of the age of onset on visual cortical responses 
$[15,22]$, while others showed that juvenile-macular degeneration patients with earlier disease forms have stronger cortical activation than age-related macular degeneration patients with later onset age [19]. Future studies should address the discrimination between age-dependency of neuroplasticity and disease-duration effects, which are separable. Disease onset age is often difficult to determine, which can make this an imprecise measure of RP severity $[11,43]$.

Given the evidence for cortical reorganization in our prior study [6], remodeling at the retinal level is unlikely. This issue can be further clarified in the future by explicitly computing population receptive fields or alternatively running experiments with artificial scotomata.

Our results did not find evidence for visual cortical structural alterations in this cohort of RP patients, showing that the visual loss level was not sufficient to produce significant cortical atrophy in the visual areas studied (V1 to V3). To our knowledge, few structural MRI studies have been conducted with low vision RP patients [3,44]. In our study, the RP patients did not present RNFL thickness atrophy which is in line with the preservation of visual cortical thickness. Nonetheless, in more advanced stages of RP disease with larger photoreceptor loss and retinal ganglion cell degeneration, disuse-driven mechanisms may lead to the visual cortical atrophy pattern that is often seen in macular degeneration, glaucoma, and also late-blindness.

\section{Conclusion}

We found that cortical visual areas (V1, V2, and V3) responses under attentional demands were increased in patients with larger degeneration of visual field. Moreover, activation during the task condition was increased for patients in both cortical regions corresponding to the preserved (FPZ) and the damaged visual field (LPZ), specifically for patients with severe visual field loss. These findings were identified in the presence of preserved visual cortical structure. The age of onset of the disease did not seem to be associated with visual cortical alterations. We conclude that RP patients may have relatively preserved visual cortical responses due to feedback attentional modulation in the absence of cortical atrophy, despite their retinal degeneration. The unmasking of corticocortical feedback signals from higher level visual regions involved in attentional processes might explain the increased cortical responses [1]. Such unmasking might lead to activation of previously silent synapses.

These results might be considered in the context of strategies for treating retinal diseases $[21,45,46]$. This is quite relevant given previous evidence that attentional cueing improves vision restoration therapy in patients with visual field loss $[47,48]$. The role of higher-level neuronal networks [49] and their functional connectivity [50] cannot be underestimated in this context. This suggests that visual responses can be dynamically adapted as a function of flexible mechanisms requiring the interaction between high-level regions that implement attentional control.

\author{
Abbreviations \\ RP: $\quad$ Retinitis Pigmentosa \\ MRI: Magnetic resonance imaging \\ fMRI: Functional magnetic resonance imaging \\ RNFL: Retinal nerve Fiber layer \\ LPZ: Lesion projection zone \\ FPZ: Function projection zone \\ deg: Degrees.
}

\section{Data Availability}

The data used to support the findings of this study are available from the corresponding author upon request.

\section{Disclosure}

This work was presented in an abstract form in the 2017 European Association for Vision and Eye Research Conference.

\section{Conflicts of Interest}

The authors declare that no conflicts of interest exist.

\section{Acknowledgments}

This work was supported by the Portuguese Funding Agency for Science and Technology (FCT) grants E-Rare2SAU/0001/2008, E-Rare4/0001/2012, COMPETE, POCI-010145-FEDER-007440, FCT. UID/NEU/04539/2013-2020, MEDPERSYST, POCI-01-0145-FEDER-016428, and BIGDATIMAGE, CENTRO-01-0145-FEDER-000016 financed by Centro 2020 FEDER, COMPETE. We would like to thank Carlos Ferreira, João Marques, and Sónia Afonso for technical assistance in the magnetic resonance acquisitions. We express our thanks to the patients and their families and the other participants for their collaboration.

\section{References}

[1] H. A. Baseler, A. Gouws, and A. B. Morland, "The organization of the visual cortex in patients with scotomata resulting from lesions of the central retina," Neuro-Ophthalmology, vol. 33, no. 3, pp. 149-157, 2009.

[2] S.-H. Cheung and G. E. Legge, "Functional and cortical adaptations to central vision loss," Visual Neuroscience, vol. 22, no. 2, pp. 187-201, 2005.

[3] D. Prins, S. Hanekamp, and F. W. Cornelissen, "Structural brain MRI studies in eye diseases: are they clinically relevant? A review of current findings," Acta Ophthalmologica, vol. 94, no. 2, pp. 113-121, 2016.

[4] K. Burnat, "Are visual peripheries forever young?," Neural Plasticity, vol. 2015, Article ID 307929, 13 pages, 2015.

[5] B. Wang, H. Yamamoto, J. Wu, and Y. Ejima, "Visual field maps of the human visual cortex for central and peripheral vision," Neuroscience and Biomedical Engineering, vol. 1, no. 2, pp. 102-110, 2013.

[6] S. Ferreira, A. C. Pereira, B. Quendera, A. Reis, E. D. Silva, and M. Castelo-Branco, "Primary visual cortical remapping in 
patients with inherited peripheral retinal degeneration," NeuroImage: Clinical, vol. 13, pp. 428-438, 2017.

[7] A. M. Rosa, M. F. Silva, S. Ferreira, J. Murta, and M. Castelo-Branco, "Plasticity in the human visual cortex: an ophthalmology-based perspective," BioMed Research International, vol. 2013, Article ID 568354, 13 pages, 2013.

[8] L. B. Merabet and A. Pascual-Leone, "Neural reorganization following sensory loss: the opportunity of change," Nature Reviews. Neuroscience, vol. 11, no. 1, pp. 44-52, 2010.

[9] A. Pascual-Leone, A. Amedi, F. Fregni, and L. B. Merabet, "The plastic human brain cortex," Annual Review of Neuroscience, vol. 28, no. 1, pp. 377-401, 2005.

[10] B. A. Wandell and S. M. Smirnakis, "Plasticity and stability of visual field maps in adult primary visual cortex," Nature Reviews Neuroscience, vol. 10, no. 12, pp. 873-884, 2009.

[11] C. Hamel, "Retinitis pigmentosa," Orphanet Journal of Rare Diseases, vol. 1, no. 1, article 40, 2006.

[12] M. A. Musarella and I. M. MacDonald, "Current concepts in the treatment of retinitis pigmentosa," Journal of Ophthalmology, vol. 2011, Article ID 753547, 8 pages, 2011.

[13] Y. Masuda, H. Horiguchi, S. O. Dumoulin et al., "Task-dependent V1 responses in human retinitis pigmentosa," Investigative Ophthalmology \& Visual Science, vol. 51, no. 10, pp. 5356-5364, 2010.

[14] E. Goesaert, M. Van Baelen, W. Spileers, J. Wagemans, and H. P. Op de Beeck, "Visual space and object space in the cerebral cortex of retinal disease patients," PLoS One, vol. 9, no. 2, article e88248, 2014.

[15] C. I. Baker, D. D. Dilks, E. Peli, and N. Kanwisher, "Reorganization of visual processing in macular degeneration: replication and clues about the role of foveal loss," Vision Research, vol. 48, no. 18, pp. 1910-1919, 2008.

[16] C. I. Baker, E. Peli, N. Knouf, and N. G. Kanwisher, "Reorganization of visual processing in macular degeneration," The Journal of Neuroscience, vol. 25, no. 3, pp. 614-618, 2005.

[17] D. D. Dilks, C. I. Baker, E. Peli, and N. Kanwisher, "Reorganization of visual processing in macular degeneration is not specific to the "preferred retinal locus"," The Journal of Neuroscience, vol. 29, no. 9, pp. 2768-2773, 2009.

[18] D. D. Dilks, J. B. Julian, E. Peli, and N. Kanwisher, "Reorganization of visual processing in age-related macular degeneration depends on foveal loss," Optometry and Vision Science, vol. 91, no. 8, pp. e199-e206, 2014.

[19] T. Liu, S. H. Cheung, R. A. Schuchard et al., "Incomplete cortical reorganization in macular degeneration," Investigative Ophthalmology \& Visual Science, vol. 51, no. 12, pp. 68266834, 2010.

[20] E. H. Schumacher, J. A. Jacko, S. A. Primo et al., "Reorganization of visual processing is related to eccentric viewing in patients with macular degeneration," Restorative Neurology and Neuroscience, vol. 26, no. 4-5, pp. 391-402, 2008.

[21] H. A. Baseler, A. Gouws, M. D. Crossland et al., "Objective visual assessment of antiangiogenic treatment for wet agerelated macular degeneration," Optometry and Vision Science, vol. 88, no. 10, pp. 1255-1261, 2011.

[22] H. A. Baseler, A. Gouws, K. V. Haak et al., "Large-scale remapping of visual cortex is absent in adult humans with macular degeneration," Nature Neuroscience, vol. 14, no. 5, pp. 649655, 2011.

[23] J. Lešták, J. Tintěra, I. Karel, Z. Svatá, and P. Rozsíval, “Functional magnetic resonance imaging in patients with the wet form of age-related macular degeneration," Neuro-Ophthalmology, vol. 37, no. 5, pp. 192-197, 2013.

[24] Y. Masuda, S. O. Dumoulin, S. Nakadomari, and B. A. Wandell, "V1 projection zone signals in human macular degeneration depend on task, not stimulus," Cerebral Cortex, vol. 18, no. 11, pp. 2483-2493, 2008.

[25] J. S. Sunness, T. Liu, and S. Yantis, "Retinotopic mapping of the visual cortex using functional magnetic resonance imaging in a patient with central scotomas from atrophic macular degeneration," Ophthalmology, vol. 111, no. 8, pp. 15951598, 2004.

[26] T. Plank, J. Frolo, S. Brandl-Rühle, A. B. Renner, H. Jägle, and M. W. Greenlee, "fMRI with central vision loss: effects of fixation locus and stimulus type," Optometry and Vision Science, vol. 94, no. 3, pp. 297-310, 2017.

[27] B. A. Wandell, S. O. Dumoulin, and A. A. Brewer, "Visual field maps in human cortex," Neuron, vol. 56, no. 2, pp. 366-383, 2007.

[28] R. O. Duncan and G. M. Boynton, "Cortical magnification within human primary visual cortex correlates with acuity thresholds," Neuron, vol. 38, no. 4, pp. 659-671, 2003.

[29] H. Strasburger, I. Rentschler, and M. Jüttner, "Peripheral vision and pattern recognition: a review," Journal of Vision, vol. 11 , no. 5 , p. 13, 2011.

[30] O. C. d'Almeida, C. Mateus, A. Reis, M. M. Grazina, and M. Castelo-Branco, "Long term cortical plasticity in visual retinotopic areas in humans with silent retinal ganglion cell loss," NeuroImage, vol. 81, pp. 222-230, 2013.

[31] J. F. Maya-Vetencourt and M. Caleo, "Insights into visual cortex plasticity: interaction between genes and sensory experience," in Visual Cortex - Current Status and Perspectives, InTech, 2012.

[32] P. Vincent-Lamarre, M. Lynn, and J. C. Béique, "The eloquent silent synapse," Trends in Neurosciences, vol. 41, no. 9, pp. 557-559, 2018.

[33] S. A. Engel, A. B. Morland, and K. V. Haak, "Plasticity, and its limits, in adult human primary visual cortex," Multisensory Research, vol. 28, no. 3-4, pp. 297-307, 2015.

[34] N. Sabbah, N. Sanda, C. N. Authié et al., "Reorganization of early visual cortex functional connectivity following selective peripheral and central visual loss," Scientific Reports, vol. 7, no. 1, article 43223, 2017.

[35] R. O. Duncan, P. A. Sample, R. N. Weinreb, C. Bowd, and L. M. Zangwill, "Retinotopic organization of primary visual cortex in glaucoma: a method for comparing cortical function with damage to the optic disk," Investigative Ophthalmology of Visual Science, vol. 48, no. 2, pp. 733-744, 2007.

[36] R. O. Duncan, P. A. Sample, R. N. Weinreb, C. Bowd, and L. M. Zangwill, "Retinotopic organization of primary visual cortex in glaucoma: comparing fMRI measurements of cortical function with visual field loss," Progress in Retinal and Eye Research, vol. 26, no. 1, pp. 38-56, 2007.

[37] K. N. Engin, B. Yemişci, S. T. Bayramoğlu et al., "Structural and functional evaluation of glaucomatous neurodegeneration from eye to visual cortex using 1.5T MR imaging: a pilot study," Journal of Clinical \& Experimental Ophthalmology, vol. 5, no. 3, p. 341, 2014.

[38] J. Lešták and J. Tintěra, "Changes in the visual cortex in patients with high-tension glaucoma," Journal of Clinical \& Experimental Ophthalmology, vol. 1, Supplement 4, p. 002, 2011. 
[39] G. Qing, S. Zhang, B. Wang, and N. Wang, "Functional MRI signal changes in primary visual cortex corresponding to the central normal visual field of patients with primary openangle glaucoma," Investigative Ophthalmology \& Visual Science, vol. 51, no. 9, pp. 4627-4634, 2010.

[40] V. M. Borges, H. V. Danesh-Meyer, J. M. Black, and B. Thompson, "Functional effects of unilateral open-angle glaucoma on the primary and extrastriate visual cortex," Journal of Vision, vol. 15, no. 15, pp. 9-14, 2015.

[41] A. M. Rosa, Â. C. Miranda, M. M. Patrício et al., "Functional magnetic resonance imaging to assess neuroadaptation to multifocal intraocular lenses," Journal of Cataract \& Refractive Surgery, vol. 43, no. 10, pp. 1287-1296, 2017.

[42] A. M. Rosa, Â. C. Miranda, M. Patrício et al., "Functional magnetic resonance imaging to assess the neurobehavioral impact of dysphotopsia with multifocal intraocular lenses," Ophthalmology, vol. 124, no. 9, pp. 1280-1289, 2017.

[43] D. T. Hartong, E. L. Berson, and T. P. Dryja, "Retinitis pigmentosa," The Lancet, vol. 368, no. 9549, pp. 1795-1809, 2006.

[44] A. Rita Machado, A. Carvalho Pereira, F. Ferreira et al., "Structure-function correlations in retinitis pigmentosa patients with partially preserved vision: a voxel-based morphometry study," Scientific Reports, vol. 7, no. 1, article 11411, 2017.

[45] M. Ashtari, L. L. Cyckowski, J. F. Monroe et al., "The human visual cortex responds to gene therapy-mediated recovery of retinal function," Journal of Clinical Investigation, vol. 121, no. 6, pp. 2160-2168, 2011.

[46] M. Ashtari, H. Zhang, P. A. Cook et al., "Plasticity of the human visual system after retinal gene therapy in patients with Leber's congenital amaurosis," Science Translational Medicine, vol. 7, no. 296, article 296ra110, 2015.

[47] D. A. Poggel, E. Kasten, and B. A. Sabel, "Attentional cueing improves vision restoration therapy in patients with visual field defects," Neurology, vol. 63, no. 11, pp. 2069-2076, 2004.

[48] D. A. Poggel, E. Kasten, E. M. Müller-Oehring, U. Bunzenthal, and B. A. Sabel, "Improving residual vision by attentional cueing in patients with brain lesions," Brain Research, vol. 1097, no. 1, pp. 142-148, 2006.

[49] B. A. Sabel, P. Henrich-Noack, A. Fedorov, and C. Gall, "Chapter 13 - Vision restoration after brain and retina damage: the "residual vision activation theory"," Progress in Brain Research, vol. 192, pp. 199-262, 2011.

[50] M. Bola, C. Gall, C. Moewes, A. Fedorov, H. Hinrichs, and B. A. Sabel, "Brain functional connectivity network breakdown and restoration in blindness," Neurology, vol. 83, no. 6, pp. 542-551, 2014. 


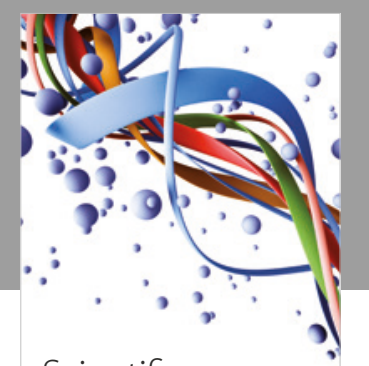

Scientifica
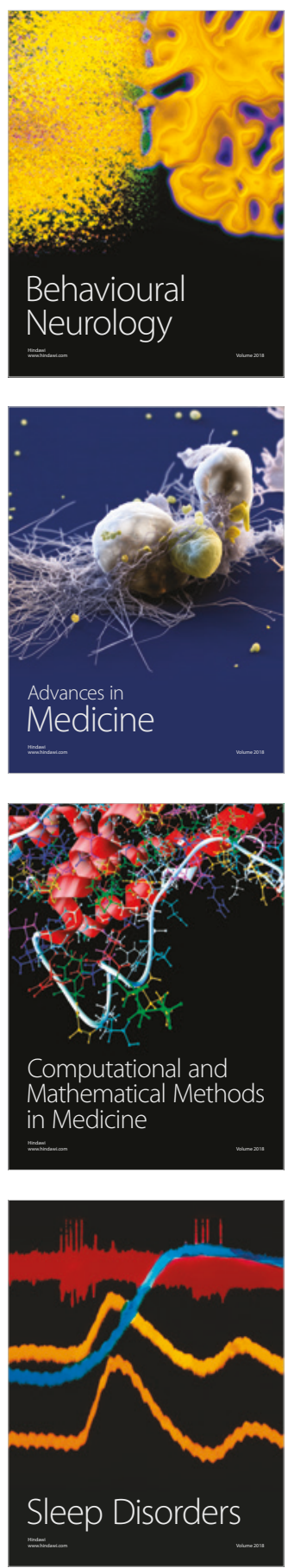

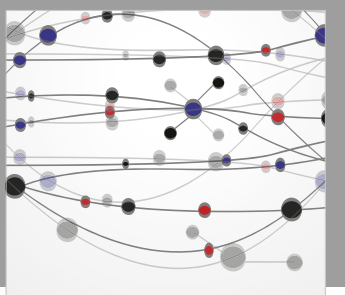

The Scientific World Journal

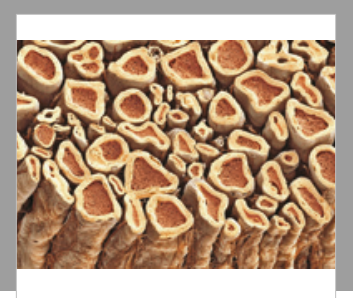

Case Reports in

Neurological Medicine

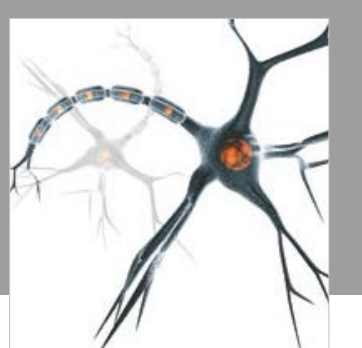

Neural Plasticity

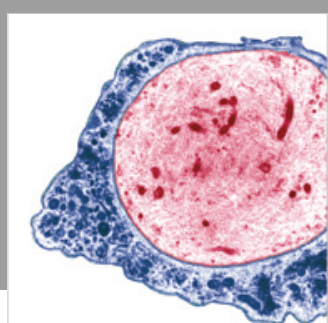

Multiple Sclerosis

International

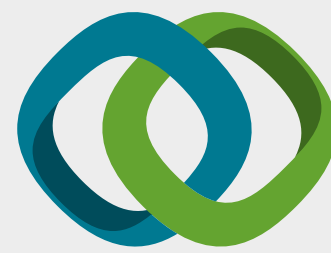

Hindawi

Submit your manuscripts at

www.hindawi.com
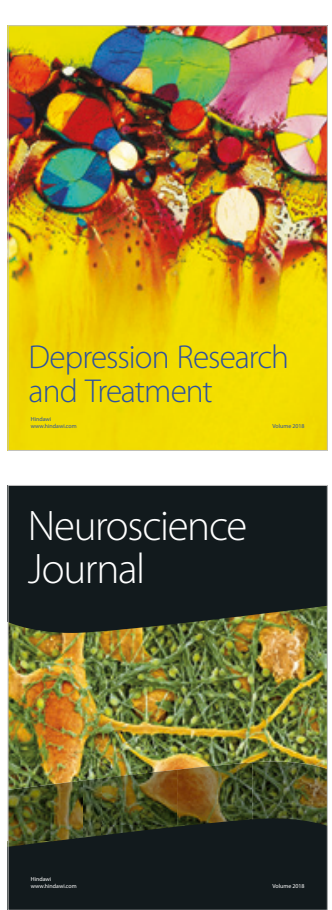

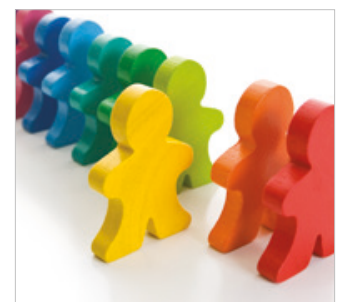

Autism

Research and Treatment
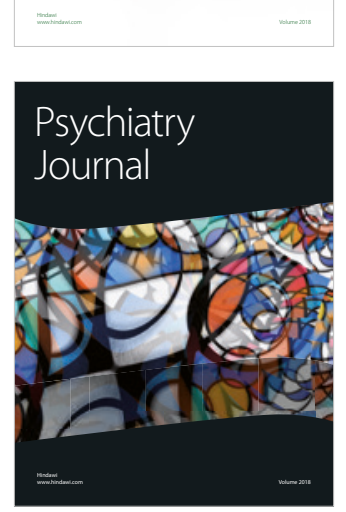
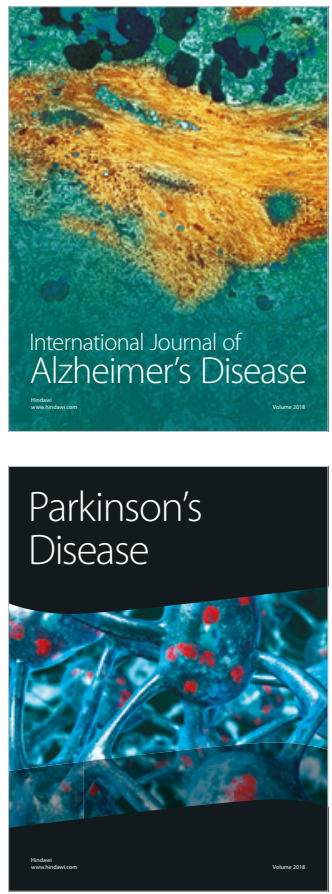
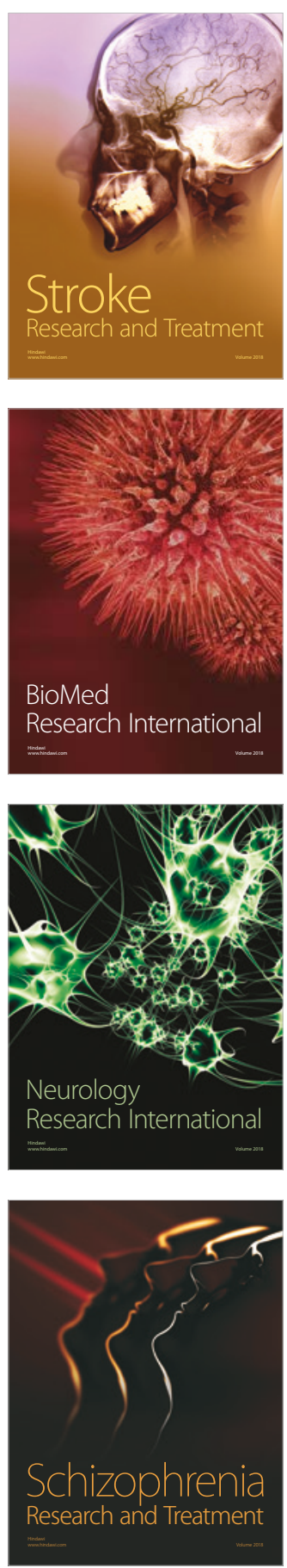